DNA DAMAGE RESPONSE

\section{Restricting repair}

different
E3 ligases
and DUBs
[cooperate]
to provide
multilayered
control of
ubiquitylation
during the
DNA damage
response

Ubiquitin signalling has a key role in maintaining genome integrity, for example by promoting the recruitment of repair proteins to DNA double-strand breaks (DSBs). However, the accumulation of ubiquitin conjugates has to be tightly regulated to avoid unscheduled repair events elsewhere in the genome. Two studies provide new insights into the mechanisms by which ubiquitylation is limited during the DNA damage response, highlighting important roles for the de-ubiquitylating enzyme (DUB) $\mathrm{POH} 1$ and the E3 ubiquitin ligases TRIP12 and UBR5.

During DNA repair, the E3 ligase RNF8 is recruited to sites of DSBs and, together with UBC13, it ubiquitylates histones $\mathrm{H} 2 \mathrm{~A}$ and $\mathrm{H} 2 \mathrm{AX}$, leading to the recruitment of the E3 ligase RNF168, which enhances the repair signal through an autoamplification mechanism. This ultimately leads to the recruitment of 53BP1, which induces repair through non-homologous end-joining (NHEJ). RNF8 is also

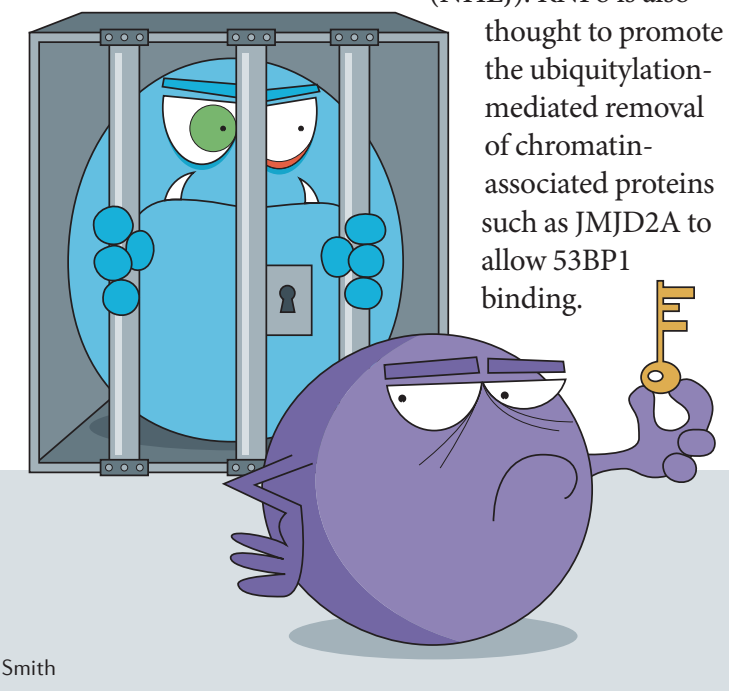

Previous studies had reported that the DUBs BRCC36, USP3 and USP16 have a role in modulating the DNA damage response at DSBs. Given the complexity of this process, however, Butler et al. speculated that other DUBs might also be involved. Indeed, a small interfering RNA screen identified the proteasomal DUB POH1 as a likely candidate. Further analysis revealed that depletion of POH1 led to an increase in the size and number of ubiquitin conjugates both at steady state as well as after exposure to hydroxyurea or ionizing radiation.

Next, Butler et al. asked which part of the DNA damage response pathway is antagonized by $\mathrm{POH} 1$. They found that $\mathrm{POH} 1$ restricts the accumulation of 53BP1 by removing Lys63 polyubiquitin chains. Lys63 polyubiquitin can be generated by RNF8 and RNF168; consistent with this, POH1 depletion restored 53BP1 accumulation in cells lacking RNF8 or RNF168, which suggests that these ligases antagonize $\mathrm{POH} 1$ function during 53BP1 recruitment to DNA damage foci. The authors also observed that $\mathrm{POH} 1$ restricts 53BP1 accumulation by promoting the maintenance of JMJD2A on chromatin. Importantly, although depletion of 53BP1, RNF8 or RNF168 reduced NHEJ, depletion of POH1 together with any of these factors increased NHEJ. Intriguingly, depletion of POH1 alone reduced NHEJ. The authors conclude that $\mathrm{POH} 1$ acts to restrict 53BP1 levels at damage foci and thereby to regulate NHEJ.

Ubiquitylation needs to be confined to DNA lesions to avoid potential deleterious consequences, but how this is achieved was not clear. In the second study,
Gudjonsson et al. carried out a screen for candidate enzymes that limit the damage response and found that the E3 ligases TRIP12 and UBR5 suppress RNF168 function during this process. Indeed, knockdown of these enzymes gave rise to large nuclear bodies containing ubiquitin that were not restricted to damage sites. Moreover, when exposed to ionizing radiation, cells depleted of TRIP12 and UBR5 carried significantly larger 53BP1 damage foci than wild-type cells. As expected, unrestrained chromatin ubiquitylation had functional consequences: for example, DNA repair was accelerated and $\mathrm{mRNA}$ synthesis was reduced.

So how do TRIP12 and UBR5 enforce localized ubiquitylation? Gudjonsson et al. first confirmed that TRIP12 and UBR5 both target RNF168. Consistent with this, depletion of TRIP12 and UBR5 led to a massive accumulation of RNF168 and, importantly, increased RNF168 loading onto chromatin; this ultimately enhanced recruitment of downstream effectors, such as 53BP1. By contrast, RNF8 did not expand outside its chromatin region. Thus, the authors conclude that TRIP12 and UBR5 restrict ubiquitylation of DNA lesions at the amplification step by limiting the numbers of RNF168 molecules.

Together, these studies highlight the importance of different E3 ligases and DUBs cooperating to provide multilayered control of ubiquitylation during the DNA damage response and to ensure that the repair process is spatially restricted.

Rachel David

ORIGINAL RESEARCH PAPERS Butler, L. R. et al The proteasomal de-ubiquitinating enzyme $\mathrm{POH} 1$ promotes the double-strand DNA break response. EMBO J. 21 Aug 2012 (doi:10.1038/emboj.2012.232)| Gudjonsson, T. et al. TRIP12 and UBR5 suppress spreading of chromatin ubiquitylation at damaged chromosomes. Cell 150, 697-709 (2012) FURTHER READING Ulrich, H. D. \& Walden, H. Ubiquitin signalling in DNA replication and repair. Nature Rev. Mol. Cell Biol. 11, 479-489 (2010) 